\title{
Vehicles while Monitoring Behavior of Dynamic Objects in a Forest-Steppe Area
}

\author{
Oleksandr Tymochko ${ }^{1}$, Andrii Trystan ${ }^{2}$, Andrii Berezhnyi ${ }^{3}$, Oleksandr Sotnikov $^{4}$, \\ Olha Matiushchenko ${ }^{5}$, Ihor Kryzhanivskyi ${ }^{2}$ \\ ${ }^{1}$ Department of Air Navigation and Combat Control of Aviation, Ivan Kozhedub Kharkiv National Air Force University, \\ Ukraine, timochko.alex@gmail.com \\ ${ }^{2}$ Department for the Development of Air Force Groups at the Air Force Scientific Center, Ivan Kozhedub Kharkiv National \\ Air Force University, Ukraine, andr.tristan@ gmail.com \\ ${ }^{3}$ Department of Air Navigation and Combat Control of Aviation, Ivan Kozhedub Kharkiv National Air Force University, \\ Ukraine, mog28@ukr.net \\ ${ }^{4}$ Department of Air Force Research Center, Ivan Kozhedub Kharkiv National Air Force University, Ukraine \\ alexsot@ukr.net \\ ${ }^{5}$ Department of Air Navigation and Combat Control of Aviation, Ivan Kozhedub Kharkiv National Air Force University,
}

\begin{abstract}
The flight route planning method for an unmanned aerial vehicle (UAV) to search for a dynamic object in a forest-steppe area was developed. Indicators and criteria of search efficiency with the participation of UAV were proposed. They allow you to choose the route that most fully meets the goals and objectives of the search. A numerical estimates of the search efficiency indicator were obtained for choosing a rational UAV flight route under conditions of uncertain behavior of a dynamic object.
\end{abstract}

Key words: Unmanned aerial vehicle, route planning, search for dynamic objects, search operation, forest-steppe area.

\section{INTRODUCTION}

Existing methods of searching for a dynamic objects using UAV are ineffective in conditions of uncertainty about its possible actions. Probabilistic estimates of dynamic objects search results are not accurate enough. Known methods for substantiating routes and methods for searching for a dynamic objects in a forest-steppe area using UAV do not correspond to methods for predicting their actions. Therefore, the use of UAV does not provide timely detection and recognition of dynamic objects in the conditions of uncertainty of their actions.

Planning a UAV route in a forest-steppe area requires the identification and consideration of special patterns in the actions of a dynamic object. While the search plan should provide for outrunning a dynamic object in actions.

\subsection{Problem analysis}

Forest-steppe terrain is characterized by significant scattered wooded areas suitable for masking search objects. The task of planning the use of UAVs for their search in such conditions and compiling the optimal flight route has not been considered. For the development of the corresponding method, the base works were [2, 6, 8, 11, 12].

Thus, the purpose of the article is to develop the flight route planning method of unmanned aerial vehicles while monitoring behavior of dynamic objects in a forest-steppe area.

\section{MAIN MATERIAL}

2.1 Performance indicators and criteria of search dynamic object using UAV in the forest-steppe area

The method is based on the principle of rationality of actions of two opposite sides. Each of the them, when choosing the routes of movement, seeks to achieve opposite goals. Dynamic objects try to reduce the likelihood of their detection on the movement paths to the target. And the search side (UAV) seeks to increase this probability. Therefore, indicators and search criteria using UAV are divided into two groups. The first group determines the efficiency of the tasks performed by the dynamic object, and the second group determines the efficiency of the UAV task.

On each $\mathrm{j}_{\mathrm{k}}-$ th route of movement of a dynamic object in the direction of the $\mathrm{k}$-th $(\mathrm{k}=1,2, \ldots, \mathrm{K})$ impact object, all open sections $i_{k}=1,2, \ldots, I_{k}$, are determined, on which UAV search is possible.

At the same time, a route efficiency indicator is introduced as the degree of vulnerability of groups of dynamic objects on the movement route $\left(\mathrm{U}_{\mathrm{jk}}\right)$

$$
\mathrm{U}_{\mathrm{jk}}=\sum_{\mathrm{i}=1}^{\mathrm{I}_{\mathrm{k}}} \mathrm{r}_{\mathrm{ijk}} \times \frac{\mathrm{T}_{\text {part_ijk }}}{\Delta \mathrm{t}_{\text {min }}},
$$

where $T_{\text {part_ijk }}$ is an estimate of the duration of observation of the site when it is overcome by the object of movement (i-th section on the $\mathrm{j}$-th route to the k-th target); 
$\Delta \mathrm{t}_{\min }$ is an estimation of the minimum required observation time for identification of an object from the air;

$r_{i j k}$ is a coefficient equal to one, if the section is included in the analysis of a possible search, and zero - otherwise.

Let there be sections on routes with nonzero vulnerability of a dynamic object (1). Then it is possible to rank routes according to the degree of vulnerability (degree of search for groups of dynamic objects).

It is assumed that a dynamic object seeks to choose a route with the least accumulated degree of vulnerability. The criterion for choosing the rational route of movement of a dynamic object, it is advisable to accept the condition of not exceeding the indicator of some predetermined limit value $\left(\mathrm{U}_{\text {lit }}\right)$.

For all sections of the studied route (for masking areas and open areas), the mathematical expectation (ME) of time to overcome them by the dynamic object $\left(\mathrm{m}_{\mathrm{t}_{\mathrm{j} \mathrm{jk}}}\right)$ and the variance of this random variable $\left(\mathrm{D}_{\mathrm{t}_{\mathrm{ijk}}}\right)$ are calculated. Let the speed of movement in the elementary section $[\mathrm{a}, \mathrm{b}]$ chosen by a dynamic object be a random variable. Let this random variable be distributed according to a uniform law.

Then you can obtain the distribution law for another random variable - the time of exit from the camouflage area (from an open area). Using the wellknown formulas of the distribution function of the random variable $(\mathrm{g}(\mathrm{t}))$, one can obtain the mathematical expectation formulas (2) of the time of movement along the section $\left(\mathrm{m}_{\mathrm{t}}\right)$ and the variance $\left(D_{t}\right)$ of this random variable $(3)$ :

$$
\begin{gathered}
m_{t}=\int_{L / b}^{L / a} t \cdot g(t) d t=\frac{L}{b-a} \cdot \ln \left(\frac{b}{a}\right) \\
D_{t}=\int_{L / b}^{L / a}\left(t-m_{t}\right)^{2} \cdot \frac{L}{t^{2}(b-a)} d t= \\
=\int_{L / b}^{L / a}\left(t-\frac{L}{b-a} \cdot \ln \left(\frac{b}{a}\right)\right)^{2} \cdot \frac{L}{t^{2}(b-a)} d t= \\
=\frac{L^{2}}{a \cdot b}-\frac{L^{2}}{(b-a)^{2}} \cdot \ln \left(\frac{b}{a}\right)^{2}, \quad \text { if } \quad b>a, \quad L=L_{M A(O A)}
\end{gathered}
$$

From all $\mathrm{J}_{\mathrm{K}}$ routes of the dynamic object to the $\mathrm{k}-\mathrm{th}$ object, one needs to be selected that better meets the criterion assessment requirement.

In total, $\mathbf{K}$ routes will be selected by the number of objects. Using formula (9), we find on these routes the total number $\mathrm{R}$ of open areas of the terrain over which UAV must fly while groups of dynamic objects are on it to detect them.
In total, $\mathbf{K}$ routes will be selected by the number of objects. Using formula (9), we find on these routes the total number $\mathbf{R}$ of open areas of the terrain over which UAV must fly to detect groups of dynamic objects during their movement in these areas.

Consider each $\mathrm{s}-\mathrm{th}(\mathrm{s}=1,2, \ldots, \mathrm{S})$ possible UAV flight route of open sections. Then each UAV flight span event will give an increase in the search efficiency indicator for each of them. This efficiency gain is denoted as $\Delta P_{\text {k.recon_n }}$, where $n=1,2, \ldots, N$ is the number of the object along the route to which the $\mathrm{k}$-th section is located. Then, as an indicator of the efficiency $\left(E_{s}\right)$ of the UAV flight route, one can use the estimate of the weighted ME of the number of dynamic object detections on the s-th UAV flight route (4).

$$
\mathrm{E}_{\mathrm{s}}=\sum_{\mathrm{n}=1}^{\mathrm{N}}\left(\mathrm{u}_{\mathrm{n}} \times \sum_{\mathrm{k}} \Delta \mathrm{P}_{\mathrm{k} \cdot \text { recon_n }}\right),
$$

where $u_{n}$ is the weight of the importance of the impact object, to which the route of the dynamic object leads and on which the this impact object is located. The importance weight is selected on the interval $[0,1]$ according to whether the search section belongs to a specific route of movement of a dynamic object.

In this case, the criterion for choosing the best of the many possible routes for the search will be the requirement to maximize the indicator (4).

It provides a comparative assessment of the effectiveness, justification and selection of a rational route for searching for dynamic objects using UAV.

\section{METHODS AND ALGORITHMS FOR SUBSTANTIATION OF THE RATIONAL SEARCH ROUTE USING UAV}

\subsection{Automatic estimation of motion parameters when performing a task by a dynamic object}

Suppose that there are $I$ wooded areas (camouflage areas in which a dynamic object cannot be detected) in the search area. Each wooded area is characterized by geometric dimensions. Within the wooded area, the length of the route of movement $\mathrm{L}_{\mathrm{MAi}}$, where $i \in \overline{1, \mathrm{I}}$.

Suppose also that in the search area there are $\mathbf{J}$ open areas between wooded areas. Each open section also has geometric dimensions, within which the length of the route of movement along the open section $\mathrm{L}_{\mathrm{OA}}$, where $\mathrm{j} \in \overline{1, \mathrm{~J}}$.

In the search area, $\mathrm{N}$ possible impact objects from the side of the dynamic object are determined. The probability of finding a dynamic object in $\mathrm{i}$-th camouflage area is equal to $\mathrm{p}_{\mathrm{i}}^{\mathrm{I}} \mathrm{A}$. The probability that a dynamic object acts on the $\mathrm{n}$-th impact object is 
equal to $\mathrm{p}_{\mathrm{n}}^{\mathrm{OI}}, \mathrm{n}=\overline{1, \mathrm{~N}}$. We take the set of masking areas $\mathrm{S}(\mathrm{MA})$ for the vertices of the directed graph. They can have incoming and outgoing edges (vertices of the first type).

The vertices of the second type will be set of impact objects $\mathrm{M}(\mathrm{O})$, which have only incoming edges. Set of open areas between the masking areas L(OA) form the edges of the directed graph. The same open area cannot connect several pairs of vertices.

At the first stage, an unweighted graph is constructed, the number of vertices of which (the power of the set of vertices) is equal to $\mathrm{V}=\mathrm{I}+\mathrm{N}$.

The task of determining the weights of the edges of the graph is associated with performance indicators of the actions of a dynamic object. One of them is the time to reach the $n$-th target from the $\mathrm{i}$-th camouflage area. Then the weights of the edges of the graph correspond to the distance between the masking areas and the impact objects. The second indicator is advisable to choose the number of open areas along the route of a dynamic object. The fewer open areas along the route, the less likely it is to detect a dynamic object (in this case, the weights of the edges of the graph are taken equal to 1).

We apply the well-known methods for solving problems on graphs to determine the weights of edges. Then from the graph of the initial situation in the search area we get the set of possible options for the action of a dynamic object. The solution to this problem is reduced to finding the minimum path from the original vertex to all the final ones. In this case, the initial vertices correspond to camouflage areas, and the final ones correspond to impact objects. Repeating the algorithm iteratively for each initial vertex (masking area), one can obtain the shortest routes from each masking area to the target. It is convenient to represent them in the form of a matrix $\mathbf{Q}$ of the following form:

$$
\mathrm{Q}=\begin{array}{l|lll} 
& 1 & \ldots & \mathrm{I} \\
\hline 1 & \mathrm{q}_{11} & \ldots & \mathrm{q}_{1 \mathrm{I}} \\
\ldots & \ldots & \mathrm{q}_{\text {in }} & \ldots \\
\mathrm{N} & \mathrm{q}_{1 \mathrm{~N}} & \ldots & \mathrm{q}_{\mathrm{IN}}
\end{array},
$$

where $q_{\text {in }}$ is a vector that has the form:

$$
\mathrm{q}_{\mathrm{in}}=\left\{\mathrm{L}_{\mathrm{in}}, \mathrm{T}_{\mathrm{in}}, \mathrm{N}_{\mathrm{in}}, \mathrm{T}_{\mathrm{in}}^{\text {entry }}, \mathrm{T}_{\mathrm{in}}^{\text {exit }}\right\},
$$

where $L_{i n}$ is the distance between the $i-t h$ camouflage area and the $n-$ th impact object; $T_{\text {in }}$ is time of movement of a dynamic object between the ith camouflage area and the $\mathrm{n}$-th impact object; $\mathrm{N}_{\text {in }}=\left\langle 1_{\mathrm{ij}}\right\rangle, \mathrm{N}_{\text {in }} \subseteq \mathrm{L}(\mathrm{I}), \mathrm{j} \in \overline{1, J}$ is the set of edges (open areas) on the route of the dynamic object to the object; $T_{i n}^{\text {entry }}\left(l_{i j}\right)=\left\langle t_{j}^{\text {entry }}\right\rangle$ is the set of values of the entry time to open areas by a dynamic object; $\mathrm{T}_{\mathrm{in}}^{\text {exit }}\left(\mathrm{l}_{\mathrm{ij}}\right)=\left\langle\mathrm{t}_{\mathrm{j}}^{\text {exit }}\right\rangle$ is the set of values of the time, when a dynamic object leaves open areas.
Matrix (5) is a set of rational routes for the movement of a dynamic object from the starting point to each possible impact object. For further calculations, the set of all open sections along the routes of a dynamic object to all objects is taken from this matrix.

\subsection{Automated preparation of proposals for} selecting the parameters search route of the UAV

The UAV search scheduling algorithm is performed in two stages:

a) the construction of the set of possible search routes with the necessary condition of flying around all open areas, but only while the dynamic object is in open areas;

b) the selection of one of the rational UAV flight routes with the maximum indicator of search efficiency.

The compiled set of open areas is described by the graph $\mathrm{G}=(\mathrm{Q}, \mathrm{E}) . \mathrm{Q} \subset \mathrm{Q}_{\mathrm{TG}}$ is the set of vertices of the graph corresponding to the set of possible open sections of terrain on the routes of movement of a dynamic object. $E$ is the set of graph edges that are described by the distance between the sections and the UAV flight time between them. The essence of matrix sorting is to arrange matrix elements in rows and columns in a specific order. According to him, an increase in the numbers of rows and columns of the matrix corresponds to an increase in the time of the output of a dynamic object in the middle of each section. After this, it can be formally stated that the possible UAV flight options have already been built in the lines. In this case, an reachability matrix (set) of possible UAV flight routes is formed (table 1). It does not yet take into account the conditions for the temporary coordination of "arrival of UAV and arrival of a dynamic object”.

\begin{tabular}{|c|c|c|c|c|c|c|c|}
\hline & 1 & 2 & $\ldots$ & $\mathbf{n}$ & $\ldots$ & $\mathbf{N}$ & $\Delta \mathbf{P}_{\text {pa }}$ \\
\hline 1 & 0 & $\Delta \mathbf{P}_{12}$ & .. & $\Delta \mathbf{P}_{1 \mathrm{n}}$ & $\ldots$ & $\Delta \mathbf{P}_{1 \mathrm{~N}}$ & $\Delta \mathbf{P}_{1}$ \\
\hline 2 & $<0$ & 0 & . & $\Delta \mathbf{P}_{2 n}$ & $\ldots$ & $\Delta \mathbf{P}_{2 \mathrm{~N}}$ & $\Delta \mathbf{P}_{2}$ \\
\hline$\ldots$ & $<0$ & $<0$ & 0 & $\Delta \mathbf{P}_{3 n}$ & $\ldots$ & $\Delta \mathbf{P}_{3 N}$ & $\Delta \mathbf{P}_{3}$ \\
\hline $\mathbf{n}$ & $<0$ & $<0$ & $<0$ & 0 & $\ldots$ & $\Delta \mathbf{P}_{\mathrm{nv}}$ & $\Delta \mathbf{P}_{n}$ \\
\hline 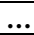 & $<0$ & $<0$ & $<0$ & $<0$ & 0 & 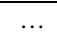 & \\
\hline $\mathbf{N}$ & $<0$ & $<0$ & $<0$ & $<0$ & $<0$ & 0 & 0 \\
\hline
\end{tabular}

Table 1: The result of sorting the UAV flight route matrix

The matrix element is a vector, which includes the time parameters (7):

$$
\begin{aligned}
& \Delta \mathrm{t}_{(\mathrm{n}-1) \mathrm{n}}=\mathrm{m}_{\mathrm{t}_{-} \mathrm{n}}-\mathrm{m}_{\mathrm{t}_{-}(\mathrm{n}-1)}>0 ; \\
& \Delta \mathrm{t}_{\mathrm{n}(\mathrm{n}-1)}=\mathrm{m}_{\mathrm{t}_{-}(\mathrm{n}-1)}-\mathrm{m}_{\mathrm{t}_{-} \mathrm{n}}<0 .
\end{aligned}
$$

Some of the matrix elements (the shaded part of the table 1) have negative values of the difference in the expected time of the dynamic object to reach the middle of the sections under consideration (due to the sorting principle), and therefore are turned off from the analysis. The second symmetric part of the matrix includes elements with positive parameters that can be 
used in search planning. In this case, several conditions are checked, one of which is advisable to write in the form (8):

$$
\mathrm{t}_{\mathrm{mn}}^{\min }-\sigma_{\mathrm{t} \_\mathrm{n}} \leq \mathrm{m}_{\mathrm{t} \_\mathrm{n}}-\mathrm{m}_{\mathrm{t} \_\mathrm{m}} \leq \mathrm{t}_{\mathrm{mn}}^{\max }+\sigma_{\mathrm{t} \_\mathrm{m}}
$$

The condition allows you to assess the compliance of UAV capabilities and requirements for the arrival of a dynamic object in the middle of the next selected section. As you move away from the first section, condition (8) will be less critical due to the growth of dispersion. Therefore, the initial sections of the search are more important for analysis.

If the next section in matrix 1 corresponds to condition (8), then it is included in the UAV flight route. For him, the required flight speed and time are calculated so that the UAV arrival and the dynamic object exit to the middle of the section are performed simultaneously. Using the spatial parameters of the section and the lowest possible UAV speed, we first calculate the time of UAV entry into the open area and departure from it (9):

$$
t_{\text {enter }_{n}}=m_{t_{n}}-\frac{L_{n}}{2 V_{U A V}^{\min }} ; t_{\text {depart }_{n}}=m_{t_{n}}+\frac{L_{n}}{2 V_{U A V}^{\min }},
$$

where $L_{n}$ is the length of the $n$-th section of the UAV flight for search. Then the probability of revealing a dynamic object in a given section $\left(\Delta \mathrm{P}_{\text {recon_n }}\right)$ can be found using tabular values of the probability integral

$$
\Delta \mathrm{P}_{\text {recon_ } \_}=\left|\mathrm{F}\left(\frac{\mathrm{t}_{\text {enter_n }}-\mathrm{m}_{\mathrm{t}_{-} \mathrm{n}}}{\sigma_{\mathrm{t}_{-} \mathrm{n}}}\right)-\mathrm{F}\left(\frac{\mathrm{t}_{\text {depart }_{-} \mathrm{n}}-\mathrm{m}_{\mathrm{t}_{-} \mathrm{n}}}{\sigma_{\mathrm{t}_{-} \mathrm{n}}}\right)\right| .
$$

Upon reaching the end of the matrix row, the generated route is included in the set of possible search routes. You can create other search routes if you start the search not from the first, but from subsequent sections. For this, an analysis of the acquisition of the search route by sections along other rows of the matrix is carried out (Table 1).

3.3 General scheme of the method for justifying the route of conducting search of dynamic objects using UAV in the forest-steppe area

The probability of detecting a dynamic object on the entire UAV flight route will allow you to select an option with a high indicator value. The resulting total probability value will characterize the mathematical expectation of the number of detections of a dynamic object along the entire UAV flight route. The decision-making method for conducting a search involving UAV allows you to select one route option, which will form the basis of the plan.

Such an approach to assessing the effectiveness of the existing method on the examples of search planning tasks in the forest-steppe area gives a value of the efficiency indicator of about 1 percent. The developed method allows to perform the same tasks of searching for a dynamic object in a forest-steppe area to obtain the numerical value of the search efficiency on a terrain with an area of 10,000 square kilometers with 18 possible impact objects. In this case, the mathematical expectation of the number of detections of a dynamic object in 56 open sections of a rational UAV flight route can be 31 .

3.4 Analysis of the results of a numerical experiment on the selection of a rational route for conducting search of UAV dynamic objects in the forest-steppe area

For a numerical experiment, forest-steppe terrain was used (Figure 1). In fig. 1 presents a graph of the terrain. Vertices 1-9 correspond to forests (camouflage areas), vertices 10-15 correspond to impact objects. We list in Table 2 the characteristics of open areas between camouflage areas. The initial search area contains nine possible masking areas, six impact objects.

Let camouflage area No. 4 be the initial area of basing of the dynamic object, and object No. 11 as the planned target (impact object). Then the probable route of movement of the dynamic object is defined as the shortest path between the vertices No. 4 and No. 11 .

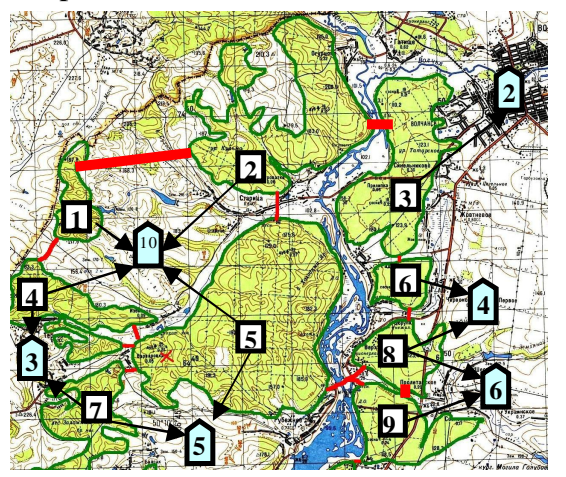

Figure 1: Map of the search area

The shortest path on the graph (Figure 2) the dynamic object will pass through the masking areas 5, 9, 8, 6, 3 . The path through the open areas will be $7.1 \mathrm{~km}$, based on the data in table 2 .

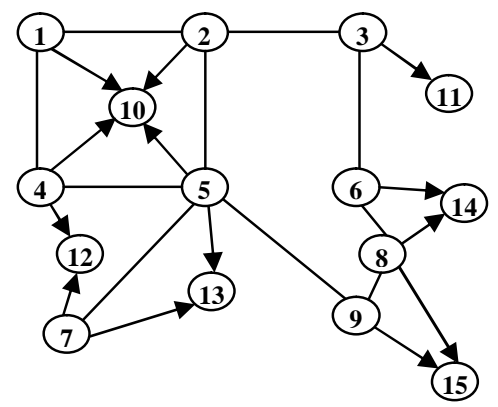

Figure 2: The graph of the formalization of the search area

Using specially developed software for modeling the movement of a dynamic object on a route, the time is estimated for the dynamic object to reach the selected impact object. 
Oleksandr Tymochko et al., International Journal of Emerging Trends in Engineering Research, 8(7), July 2020,3208 - 3215

Table 2: Characteristics of open areas in the search area

\begin{tabular}{|c|c|c|c|c|}
\hline $\begin{array}{c}\text { No } \\
\mathbf{i} / \mathbf{o}\end{array}$ & $\begin{array}{c}\text { The number of the } \\
\text { open area (graph } \\
\text { edge) }\end{array}$ & $\begin{array}{c}\text { Length, } \\
{[\mathbf{k m}]}\end{array}$ & $\begin{array}{c}\text { Minimum speed } \\
\text { dynamic object, } \\
{[\mathbf{k m} / \mathbf{h}]}\end{array}$ & $\begin{array}{c}\text { Maximum } \\
\text { speed dynamic } \\
\text { object, }[\mathbf{k m} / \mathbf{h}]\end{array}$ \\
\hline 1 & 2 & 3 & 4 & 5 \\
\hline 1 & $1-2$ & 10 & 3 & 6 \\
\hline 2 & $1-4$ & 2 & 3 & 6 \\
\hline 3 & $1-10$ & 2 & 3 & 6 \\
\hline 4 & $2-3$ & 1 & 2 & 3 \\
\hline 5 & $2-5$ & 2,5 & 3 & 4 \\
\hline 6 & $2-10$ & 8 & 3 & 5 \\
\hline 7 & $3-6$ & 0,5 & 4 & 5 \\
\hline 8 & $3-11$ & 3 & 2 & 3 \\
\hline 9 & $4-5$ & 1 & 4 & 6 \\
\hline 10 & $4-10$ & 3 & 4 & 5 \\
\hline 11 & $4-12$ & 0,6 & 3 & 5 \\
\hline 12 & $5-7$ & 0,7 & 3 & 4 \\
\hline 13 & $5-9$ & 1,2 & 1 & 2 \\
\hline 14 & $5-10$ & 3 & 2 & 6 \\
\hline 15 & $5-13$ & 1,5 & 2 & 5 \\
\hline 16 & $6-8$ & 0,8 & 3 & 5 \\
\hline 17 & $6-14$ & 2 & 2 & 6 \\
\hline 18 & $7-12$ & 1,1 & 2 & 6 \\
\hline 19 & $7-13$ & 3,5 & 2 & 6 \\
\hline 20 & $8-9$ & 0,6 & 3 & 5 \\
\hline 21 & $8-14$ & 1,7 & 2 & 6 \\
\hline 22 & $8-15$ & 2,3 & 2 & 6 \\
\hline 23 & $9-15$ & 0,9 & 2 & 6 \\
\hline
\end{tabular}

Let the speed of a dynamic object (group) be from 4 to $5 \mathrm{~km}$, distributed according to the uniform distribution law. The section is divided into 100 segments of $10 \mathrm{~m}$. The number of runs of the model is 500, which ensures statistical stability of the results. Calculations showed that with such initial data, the ME of the passage time of the section is 0.223 hours, and the standard deviation is $\sigma_{4-5}=0,014$ hours.

Similar calculations of the mathematical expectation and standard deviation for each open area along the route from the 4th camouflage area (4th vertex of the graph in Figure 5) to the 2 nd impact object (11th vertex of the graph in Figure 2) were performed. The results are presented in table 3 .

Table 3: Prediction of rout time of open sections of dynamic objects on a given route

\begin{tabular}{|c|c|c|c|}
\hline $\begin{array}{c}\text { No. } \\
\text { i/o }\end{array}$ & $\begin{array}{c}\text { Edge } \\
\text { number }\end{array}$ & $\begin{array}{c}\text { The mathematical expectation } \\
\text { of rout time, }[\mathbf{h}]\end{array}$ & $\begin{array}{c}\text { Standard deviation } \\
\text { of rout time, }[\mathbf{h}]\end{array}$ \\
\hline 1 & $4-5$ & 0,223 & 0,014 \\
\hline 2 & $5-9$ & 0,8 & 0,075 \\
\hline 3 & $8-9$ & 0,15 & 0,015 \\
\hline 4 & $6-8$ & 0,2 & 0,018 \\
\hline 5 & $3-6$ & 0,11 & 0,006 \\
\hline 6 & $3-11$ & 1,21 & 0,012 \\
\hline
\end{tabular}

Then, the time of movement of a dynamic object in the camouflage areas on a given route is determined. The results are listed in table 4 . The length of the camouflage area is taken as the distance from the entrance of the open area to its exit (for this route).

Table 4: Prediction of the transit time of masking areas by a dynamic object on a given route

\begin{tabular}{|c|c|c|c|c|c|c|}
\hline $\begin{array}{c}\text { No } \\
\text { i/o }\end{array}$ & $\begin{array}{c}\text { MA } \\
\text { Number }\end{array}$ & $\begin{array}{c}\text { Length, } \\
{[\mathbf{k m}]}\end{array}$ & $\begin{array}{c}\text { Min. V } \\
\text { dynamic } \\
\text { object, [km/h] }\end{array}$ & $\begin{array}{c}\text { Max. V } \\
\text { dynamic } \\
\text { object, }[\mathbf{k m} / \mathbf{h}]\end{array}$ & $\begin{array}{c}\text { ME rout } \\
\text { time, [h] }\end{array}$ & $\begin{array}{c}\text { SD rout } \\
\text { time, [h] }\end{array}$ \\
\hline 1 & 4 & 3 & 2 & 3 & 1,21 & 0,02 \\
\hline 1 & 2 & 3 & 4 & 5 & 6 & 7 \\
\hline 2 & 5 & 10 & 2 & 3 & 2,84 & 0,03 \\
\hline 3 & 9 & 1,5 & 3 & 4 & 0,43 & 0,0013 \\
\hline 4 & 8 & 3 & 2 & 3 & 1,21 & 0,02 \\
\hline 5 & 6 & 1,5 & 2 & 3 & 0,6 & 0,012 \\
\hline 6 & 3 & 5 & 2 & 3 & 2,03 & 0,02 \\
\hline
\end{tabular}

We write down the results of modeling the movement of a dynamic object along the route in table 5. The time schedule of the movement of a dynamic object from the 4th masking area to the 2nd impact object (11th vertex of the graph) is shown in Figure 3.

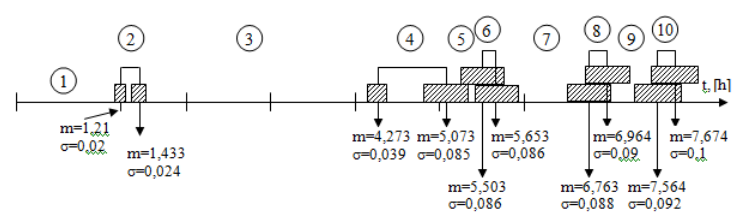

Figure 3: Timeline of the movement of dynamic objects along the route No. 4 - No. 11

In figure 3 , the numbers in the circle show the number of the route section corresponding to the line in table 5 .

Table 5: Prediction of the rout time of dynamic object along a route (No.4-No.11)

\begin{tabular}{|c|c|c|c|c|c|c|c|}
\hline $\begin{array}{c}\text { No } \\
\text { i/o }\end{array}$ & $\begin{array}{c}\text { Route } \\
\text { section }\end{array}$ & $\begin{array}{c}\text { ME of } \\
\text { entry } \\
\text { time, } \\
{[\mathbf{h}]}\end{array}$ & $\begin{array}{c}\text { SD, } \\
{[\mathbf{h}]}\end{array}$ & $\begin{array}{c}\mathbf{3}^{* \mathbf{S D},} \\
{[\mathbf{h}]}\end{array}$ & $\begin{array}{c}\text { ME of } \\
\text { exit } \\
\text { time, } \\
{[\mathbf{h}]}\end{array}$ & $\begin{array}{c}\text { SD, } \\
{[\mathbf{h}]}\end{array}$ & $\begin{array}{c}\mathbf{3}^{* \mathbf{S}} \\
\mathbf{D ,} \\
{[\mathbf{h}]}\end{array}$ \\
\hline 1 & MA №4 & 0 & 0 & 0 & 1,21 & 0,02 & 0,06 \\
\hline 2 & OA №4-5 & 1,21 & 0,02 & 0,06 & 1,433 & 0,024 & 0,084 \\
\hline 3 & MA №5 & 1,433 & 0,024 & 0,084 & 4,273 & 0,039 & 0,117 \\
\hline 4 & OA №5-9 & 4,273 & 0,039 & 0,117 & 5,073 & 0,085 & 0,255 \\
\hline 5 & MA №9 & 5,073 & 0,085 & 0,255 & 5,503 & 0,085 & 0,255 \\
\hline 6 & OA №9-8 & 5,503 & 0,085 & 0,255 & 5,653 & 0,086 & 0,258 \\
\hline 7 & MA №8 & 5,653 & 0,086 & 0,258 & 6,763 & 0,088 & 0,264 \\
\hline 8 & OA №8-6 & 6,763 & 0,088 & 0,264 & 6,964 & 0,09 & 0,27 \\
\hline 9 & MA №6 & 6,964 & 0,09 & 0,27 & 7,564 & 0,092 & 0,276 \\
\hline 10 & OA №6-3 & 7,564 & 0,092 & 0,276 & 7,674 & 0,1 & 0,3 \\
\hline 11 & MA №3 & 7,674 & 0,1 & 0,3 & 9,704 & 0,11 & 0,33 \\
\hline
\end{tabular}

Similarly, the time of movement of a dynamic object along the route is predicted when it moves from the fourth masking area to the 4 th, 5th and 6th impact objects $(14,13,15$ vertices of the graph, respectively). Applying Dijkstra's algorithm for an oriented weighted graph (Figure 4), the routes of the movement of a dynamic object from the 4th masking area to the 4th, 5th and 6th impact objects, respectively, are obtained. Using the data from table 2 and table 4 , tables 6-8 are filled in, similar to table 5 (forecast of the time of movement of a dynamic object along a route).

Timelines of the movement of a dynamic object for each route are presented in Figure 4. Its analysis showed that when initially located in the 4th camouflage area, the search for a dynamic object should be performed in an open area 4-5. At the same time, the UAV flight time over an open area for guaranteed location of a dynamic object on it should be $t_{\text {min }}=1,21+0,06=1,27$ hours from the moment the dynamic object is detected to $t_{\max }=1,433+0,084=1,349$ the time it leaves the open area. Thus, the guaranteed time of a dynamic object in an open area will be 0.08 hours (about 5 minutes). This is enough to detect a dynamic object using UAV. 
Oleksandr Tymochko et al., International Journal of Emerging Trends in Engineering Research, 8(7), July 2020,3208 - 3215

Table 6: Prediction of the rout time of dynamic object along a route (No.4-No.14)

\begin{tabular}{|c|c|c|c|c|c|c|c|}
\hline $\begin{array}{c}\text { № } \\
\text { i/o }\end{array}$ & Route section & $\begin{array}{c}\text { ME of } \\
\text { entry time, } \\
{[\mathbf{h}]}\end{array}$ & $\mathbf{S D},[\mathbf{h}]$ & $\begin{array}{c}3 * \text { SD, } \\
{[\mathbf{h}]}\end{array}$ & $\begin{array}{c}\text { ME of } \\
\text { exit time, } \\
{[\mathbf{h}]}\end{array}$ & $\mathbf{S D},[\mathbf{h}]$ & $\begin{array}{c}3 * \mathbf{S D}, \\
{[\mathbf{h}]}\end{array}$ \\
\hline 1 & MA №4 & 0 & 0 & 0 & 1,21 & 0,02 & 0,06 \\
\hline 2 & OA №4-5 & 1,21 & 0,02 & 0,06 & 1,433 & 0,024 & 0,084 \\
\hline 3 & MA №5 & 1,433 & 0,024 & 0,084 & 4,273 & 0,039 & 0,117 \\
\hline 4 & OA №5-9 & 4,273 & 0,039 & 0,117 & 5,073 & 0,085 & 0,255 \\
\hline 5 & MA №9 & 5,073 & 0,085 & 0,255 & 5,503 & 0,085 & 0,255 \\
\hline 6 & OA №9-8 & 5,503 & 0,085 & 0,255 & 5,653 & 0,086 & 0,258 \\
\hline 7 & MA №8 & 5,653 & 0,086 & 0,258 & 6,763 & 0,088 & 0,264 \\
\hline 8 & OA №8-14 & 6,763 & 0,88 & 0,264 & 7,233 & 0,09 & 0,27 \\
\hline
\end{tabular}

Table 7: Prediction of the rout time of dynamic object along a route (No.4-No.13)

\begin{tabular}{|c|c|c|c|c|c|c|c|}
\hline $\begin{array}{c}\text { № } \\
\text { i/o }\end{array}$ & $\begin{array}{c}\text { Route } \\
\text { section }\end{array}$ & $\begin{array}{c}\text { ME of entry } \\
\text { time, [h] }\end{array}$ & SD, [h] & $*$ SD, [h] & $\begin{array}{c}\text { ME of } \\
\text { exit } \\
\text { me, [h] }\end{array}$ & SD, [h] & * $*$ SD, [h] \\
\hline 1 & MA №4 & 0 & 0 & 0 & 1,21 & 0,02 & 0,06 \\
\hline 2 & OA №4-5 & 1,21 & 0,02 & 0,06 & 1,433 & 0,024 & 0,084 \\
\hline 3 & MA №5 & 1,433 & 0,024 & 0,084 & 4,273 & 0,039 & 0,117 \\
\hline 4 & OA №5-13 & 4,273 & 0,039 & 0,117 & 4,733 & 0,04 & 0,12 \\
\hline
\end{tabular}

Table 8: Prediction of the rout time of dynamic object along a route (No.4-No.15)

\begin{tabular}{|c|c|c|c|c|c|c|c|}
\hline $\begin{array}{c}\text { № } \\
\text { i/o }\end{array}$ & Route section & $\begin{array}{c}\text { ME of } \\
\text { entry time, } \\
{[\mathbf{h}]}\end{array}$ & $\mathbf{S D},[\mathbf{h}]$ & $\mathbf{3} * \mathbf{S D}, \begin{array}{c}\text { ME of } \\
{[\mathbf{h}]}\end{array}$ & $\begin{array}{c}\text { exit time, } \\
{[\mathbf{h}]}\end{array}$ & $\mathbf{S D},[\mathbf{h}]$ & $\begin{array}{c}\mathbf{3} \text { SD } \\
{[\mathbf{h}]}\end{array}$ \\
\hline 1 & MA №4 & 0 & 0 & 0 & 1,21 & 0,02 & 0,06 \\
\hline 2 & OA №4-5 & 1,21 & 0,02 & 0,06 & 1,433 & 0,024 & 0,084 \\
\hline 3 & MA №5 & 1,433 & 0,024 & 0,084 & 4,273 & 0,039 & 0,117 \\
\hline 4 & OA №5-9 & 4,273 & 0,039 & 0,117 & 5,073 & 0,085 & 0,255 \\
\hline 5 & MA №9 & 5,073 & 0,085 & 0,255 & 5,503 & 0,085 & 0,255 \\
\hline 6 & OA №9-15 & 5,503 & 0,085 & 0,255 & 5,723 & 0,086 & 0,258 \\
\hline
\end{tabular}

If UAV search is not possible in the indicated open area (for example, due to the late receipt of information about the initial location of the dynamic object), the search should be carried out in the open area between camouflage areas 5-9 (Figure 4), except for the option to exit the dynamic object to the 5 th impact object.

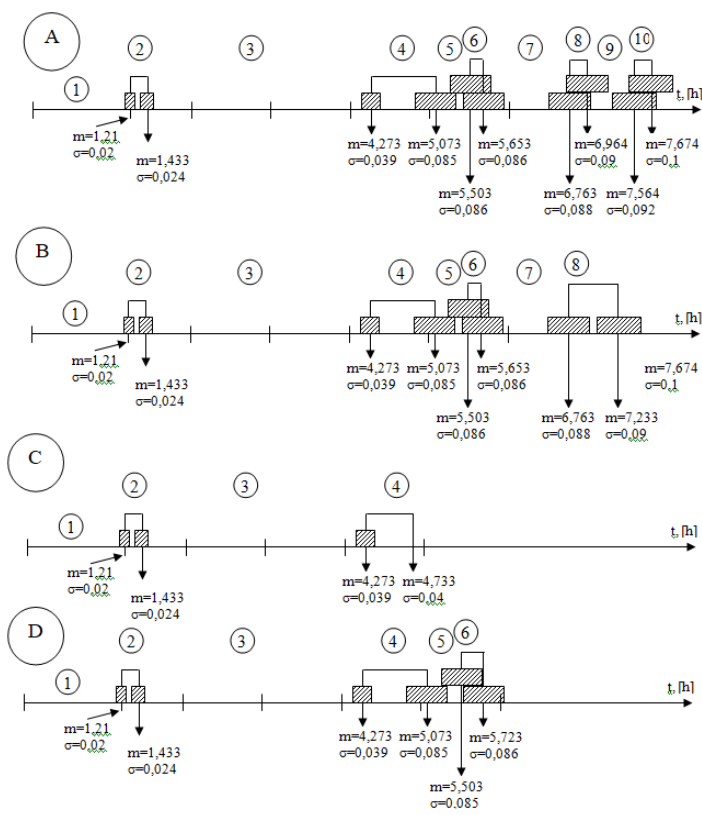

Figure 4 Timelines of dynamic object movement from the 4th camouflage area (A - route "4-11", B - route "4-14", C - route " $4-13$ ", D - route " $4-15$ ")
The time of guaranteed finding of a dynamic object in a given open area is from $\mathrm{t}_{\min }=4,273+0,039 \cdot 3=4,39$ hour from the moment of detecting a dynamic object to $\mathrm{t}_{\max }=5,073-0,085 \cdot 3=4,818$ hour - the time that the dynamic object leaves the open area.

Thus, the time guaranteed to find a dynamic object in an open area will be approximately 25 minutes. This is enough to detect a dynamic object using UAV.

Knowing that both random variables are distributed according to the normal distribution law, we find the probability of finding a dynamic object in each time interval presented on the graph.

The first time interval $[5,245 ; 5.395]$. The probability that a dynamic object is in an open area in a given time interval is equal to:

$$
\mathrm{P}_{1}=\mathrm{F}\left(\frac{5,395-5,503}{0,086}\right)-\mathrm{F}\left(\frac{5,245-5,503}{0,086}\right) \approx 0,4 .
$$

The second time interval $[5,395 ; 5,761]$. The probability of finding a dynamic object in an open area in a given time interval consists of the probability of two independent events: the probability that a dynamic object will be in a given interval $\mathrm{P}_{2}^{\text {entry }}$, and the probability that a dynamic object will not leave this open area $\mathrm{P}_{2}^{\mathrm{nl}}=1-\mathrm{P}_{2}^{\text {exit }}$.

Thus, $\mathrm{P}_{2}=\mathrm{P}_{2}^{\text {entry }} \cdot\left(1-\mathrm{P}_{2}^{\text {exit }}\right)$ :

$$
\begin{gathered}
\mathrm{P}_{2}^{\text {entry }}=\mathrm{F}\left(\frac{5,761-5,503}{0,086}\right)-\mathrm{F}\left(\frac{5,395-5,503}{0,086}\right) \approx 0,6 ; \\
\mathrm{P}_{2}^{\text {exit }}=\mathrm{F}\left(\frac{5,761-5,653}{0,086}\right)-\mathrm{F}\left(\frac{5,395-5,653}{0,086}\right) \approx 0,6 ; \\
\mathrm{P}_{2}=0,6 \cdot(1-0,6)=0,24 .
\end{gathered}
$$

The third time interval $[5,761 ; 5,911]$. The probability that a dynamic object is in an open area in a given time interval is equal to:

$$
\mathrm{P}_{3}=1-\left[\mathrm{F}\left(\frac{5,911-5,653}{0,086}\right)-\mathrm{F}\left(\frac{5,761-5,653}{0,086}\right)\right] \approx 0,6 .
$$

Thus, it is possible to determine the probability of finding a dynamic object in an open area in any time range and set the time range for viewing the open area to achieve a probability of finding at least a given one.

We take the probability of correct recognition of a dynamic object using UAV $-\mathrm{P}_{\mathrm{RC}}=0,9$, and the probability of correct identification of a ground object as a dynamic object $-\mathrm{P}_{\mathrm{ID}}=0,8$. We accept the equally probable hypothesis about the impact objects for the dynamic object, which is located in the 4th camouflage area. The results are summarized in table 9 . 
Table 9: Schedule of open area during UAV search missions

\begin{tabular}{|c|c|c|c|c|c|c|c|}
\hline $\begin{array}{c}\text { № } \\
\text { i/o }\end{array}$ & $\begin{array}{c}\text { Open Area } \\
\text { Number }\end{array}$ & $\begin{array}{c}\text { Entry } \\
\text { time }\end{array}$ & $\begin{array}{c}\text { Departure } \\
\text { time }\end{array}$ & $\begin{array}{c}\text { The probability } \\
\text { of finding } \\
\text { dynamic object } \\
\text { in an open area }\end{array}$ & PRC & PID & PAR \\
\hline 1 & OA №4-5 & $\begin{array}{c}1 \mathrm{~h} 16 \\
\mathrm{~min}\end{array}$ & $1 \mathrm{~h} 21 \mathrm{~min}$ & 1 & 0,9 & 0,8 & 0,72 \\
\hline 2 & OA №5-9 & $\begin{array}{c}4 \mathrm{~h} 25 \\
\mathrm{~min}\end{array}$ & $4 \mathrm{~h} 50 \mathrm{~min}$ & 1 & 0,9 & 0,8 & 0,72 \\
\hline 3 & OA №9-8 & $\begin{array}{c}5 \mathrm{~h} 45 \\
\mathrm{~min}\end{array}$ & $5 \mathrm{~h} 54 \mathrm{~min}$ & 0,6 & 0,9 & 0,8 & 0,432 \\
\hline 4 & OA № 8-6 & $\begin{array}{c}6 \mathrm{~h} 42 \\
\mathrm{~min}\end{array}$ & $7 \mathrm{~h} 02 \mathrm{~min}$ & 0,52 & 0,9 & 0,8 & 0,37 \\
\hline 5 & OA № 6-3 & $\begin{array}{c}7 \mathrm{~h} 22 \\
\mathrm{~min}\end{array}$ & $7 \mathrm{~h} 31 \mathrm{~min}$ & 0,6 & 0,9 & 0,8 & 0,432 \\
\hline
\end{tabular}

The probability of the UAV task in this case, when flying open areas, according to table 9, is:

$$
\mathrm{P}_{\mathrm{AR}_{4-6}}=1-(1-0,72) \cdot(1-0,72)=0,9216
$$

Thus, the probability of successful UAV surface monitoring using the proposed approach when finding a dynamic object in the fourth masking area (Figure 1) is:

$$
\begin{aligned}
& \mathrm{P}_{\mathrm{AR}}=0,25 \cdot 0,984+0,25 \cdot 0,955+0,25 \cdot 0,72+ \\
& +0,25 \cdot 0,9216=0,895 .
\end{aligned}
$$

Similarly, the search efficiency is calculated when a dynamic object is found in other masking areas (tables 10, 11).

Table 10: Optimization of the traffic routes of dynamic object to the object No. 1

\begin{tabular}{|c|c|c|c|c|c|c|c|c|}
\hline \multirow{2}{*}{$\begin{array}{c}\text { No.OA } \\
\text { No.MA }\end{array}$} & \multicolumn{8}{|c|}{\begin{tabular}{|c|c|c|}
\multirow{2}{*}{ Increase in the degree of vulnerability of dynamic } \\
object (Ujk) on traffic routes
\end{tabular}} \\
\cline { 2 - 9 } & $\mathbf{1}$ & $\mathbf{2}$ & $\mathbf{3}$ & $\mathbf{4}$ & $\mathbf{5}$ & $\mathbf{6}$ & $\mathbf{7}$ & \\
\hline \multirow{4}{*}{1} & 12,3 & 8,4 & 0 & 3,35 & 0 & 0 & 3,4 & 28,45 \\
\cline { 2 - 9 } & 12,3 & 0 & 2,34 & 3,35 & 3,2 & 0 & 0 & 21,19 \\
\cline { 2 - 9 } & 0 & 8,4 & 2,34 & 0 & 3,2 & 5,6 & 0 & 19,54 \\
\cline { 2 - 8 } & 0 & 8,4 & 2,34 & 0 & 3,2 & 5,6 & 3,4 & 22,94 \\
\cline { 2 - 8 } & 0 & 8,4 & 0 & 3,35 & 0 & 5,6 & 3,4 & 20,75 \\
\hline
\end{tabular}

Table 11: Optimization of UAV flight search routes for dynamic object in open areas of the entire set of routes of their traffic

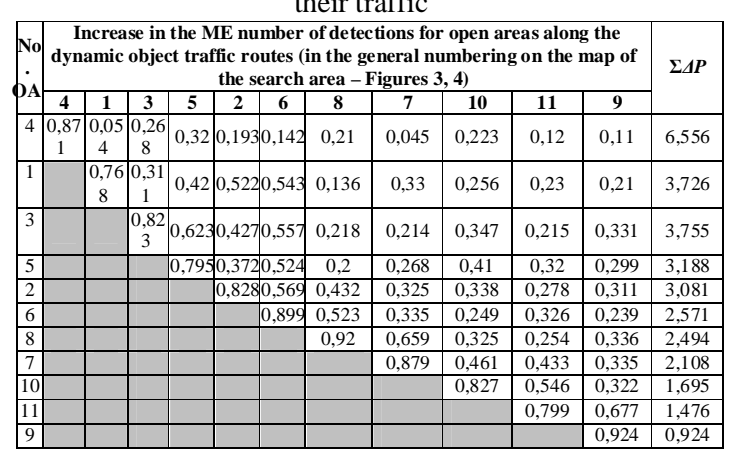

\section{CONCLUSION}

1. A flight route planning method of unmanned aerial vehicles while monitoring behavior of dynamic objects in a forest-steppe area was developed. The presented method is actually a sequence of visits to certain points to search for dynamic objects, reducing the degree of uncertainty of their actions.
2. The obtained estimates confirm the correctness of the calculations and correspond to the experimental results.

3. Further development of this method consists in expanding the planning of search routes for UAV groups, subject to their group interaction and exchange of information about detected objects.

\section{REFERENCES}

1. Shreyamsh Kamate, Nuri Yilmazer, Application of Object Detectionand Tracking Techniques for Unmanned Aerial Vehicles, Texas A\&M University - Kingsville, Kingsville, TX 78363, USA

2. Krylov I.G. Development Of Information Technology For The Organization Of The Coordinated Actions of A Group of Unmanned Aerial Vehicles In The Search For Mobile Ground Objects, diss. for the degree of Cand. tech. sciences for special. 05.13.01, Moscow Aviation University Moscow, RF, 143 p.

3. Musab Coúkuna, Sencer Ünala, Implementation of Tracking of a Moving Object Based on Camshift Approach with a UAV, Available online at www.sciencedirect.com ScienceDirect

4. George Plastiras, Christos Kyrkou, and Theocharis Theocharides, Efficient ConvNetbased Object Detection for Unmanned Aerial Vehicles by Selective Tile Processing, 2018. In Proceedings of International Conference on Distributed Smart Cameras, Eindhoven, Netherlands, September 3-4, 2018 (ICDSC'18), 6 pages.

5. O.Tymochko, A.Ttystan, V.Ushan , N.Yeromina ,O.Dmitriiev , V.Mazharov , I.Padalka , I.Hannoshyna , I.Masik , A. Zazirnyi, The Synthesis of the Reference Image and Algorithms for Vehicle Navigation Systems, JETER,8(3),2020, pp. 853 - 858. doi:10.30534/ijeter/2020/40832020

6. Yan Jin, Yan Liao, Ali A. Minai, and Marios M. Polycarpou, Balancing Search and Target Response in Cooperative Unmanned Aerial Vehicle (UAV) Teams, CYBERNETICS, VOL. 36, NO. 3, JUNE 2006.

7. Min-Hyuck Lee and Seokwon Yeom, Detection and Tracking of Multiple Moving Vehicles with a UAV, International Journal of Fuzzy Logic and Intelligent Systems Vol. 18, No. 3, September 2018, pp. 182-189

8. Joel George, Sujit P. B., J. B. Sousa, Search Strategies for Multiple UAV Search and Destroy Missions, Journal of Intelligent \& Robotic Systems January 2011, Volume 61, Issue 1-4, pp 355-367

9. Oleksandr Tymochko, Andrii Trystan, Andrii Berezhnyi, Olga Matiushchenko, Oleksandr Timochko. Method of Automated Flight Route Planning for Unmanned Aerial Vehicles to Search for Stationary Objects, In Proceedings Of 2020 IEEE 11th International 
Conference on Dependable Systems, Services and Technologies (DESSERT'2020), Ukraine, 2020. - 242-246 pp.

10. Ziyang, Z. H. E. N., Distributed intelligent self-organized mission planning of multiUAV for dynamic targets cooperative searchattack, Chinese Journal of Aeronautics, vol. 32, no. 12, pp. 2706-2716, December 2019.

11. Moiseev D.V.; Chin V.M.; Moiseeva S.G.; Fam S.K., Routing the Flight of a Light Unmanned Aerial Vehicle of Unequal Objects in a Constant Wind Field, Taking into Account the Restrictions on the Flight Time Along the Route, in Aviation and Cosmonautic, Moscov, 2015.

12. Sotnikov, A., Tarshyn, V., Yeromina N., Petrov, S., \& Antonenko, N, A Method for Localization a reference Object in a Current Image with Several Bright Objects, EasternEuropean Journal of Enterprise Technologies, 3 (87), 68-74.

13. M.Iasechko, Y. Gnusov, I. Manzhai, O. Uhrovetskyi, V.Manoylo, A. Iesipov,O. Zaitsev, M. Volk, and O. Vovk. Determination of requirements for the protection of radioelectronic equipment from the terroristic influence by electromagnetic radiation, IJETER, 7(12), 2019, pp. $772-777$. doi: 10.30534/ijeter/2019/077122019.

14. M. Iasechko, V. Larin, D. Maksiuta, O. Ochkurenko, Y.Samsonov, H. Lyashenko, A.Zinchenko, and R.Vozniak. Model description of the modified solid state plasma material for electromagnetic radiation protection, IJETER, 7(10), 2019, pp. 376- 382. doi: 10.30534/ijeter/2019/027102019.

15. O. Turinskyi, M. Burdin, M. Iasechko, V. Larin, Y. Gnusovand V. Manoylo. Protection of board radioelectronic equipment from the destructive powerful electromagnetic radiation with the use of natural technologies, IJETER, 7(11), 2019, pp. 542548. doi: 10.30534/ijeter/2019/237112019. 